\title{
Finite element analysis of Reactive Powder Concrete beam flexural properties
}

\author{
Cuiping Chi ${ }^{1, a}$, Changchun Pei ${ }^{1, b^{*}}$ \\ ${ }^{1}$ Department of Structural Engineering, College of Engineering, Yanbian University, Yanji, China

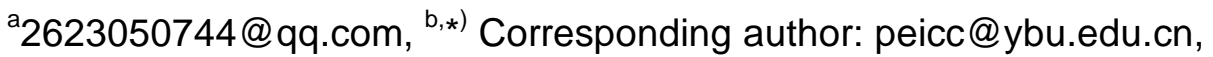

Keywords: Reactive Powder Concrete beam; Finite element analysis; combination of different fibers; flexural capacity

Abstract. By using finite element analysis to study the effects of different water-cement ratio and different fiber composition on the flexural properties of Reactive Powder Concrete beam. The results show that: Firstiy, under the same fiber combination, with the increase of water-cement ratio, the bending moment decreases gradually while the mid-span deflection gradually increases. Secondly, under the same W/ B, the fiber combination B with the best flexural strength was obtained, followed by decreasing in the order of C, A, D. In this paper, the flexural capacity of the Reactive Power Concrete beams is analyzed by finite element simulation. The flexural capacity formula of the Reactive Power Concrete beams with different fiber-reinforced is deduced.

\section{Introduction}

With the rapid development of the economy, the construction industry tends to super high, large span, large scale, and then the concrete material requirements of high strength, ultra high strength. But because of high strength, super high strength concrete with high brittleness, poor ductility due to its application was restricted. Reactive powder concrete(RPC) was first developed by France in the 1990s [1], and solved the problem effectively. RPC is a new type of cement-based composite material with high strength, low brittleness and excellent durability. It has broad application prospects, and the incorporation of fiber in RPC can make up the cracks in the concrete and improve the ductility. At present, the mechanical properties of RPC have been studied at home and abroad, and some remarkable results have been obtained [2-6]. However, the influence of fiber on the flexural behavior of RPC beams and the study of constitutive relation are relatively few.

Therefore, the influence of different water cement ratio (W/B) and different fiber combinations on the bending moment and deflection of the RPC beam under the initial crack and yield state is analyzed by using the finite element method RPC, and provides technical reference for the application of RPC beam in practical engineering.

\section{Conceptual design}

In this paper, 12 beams of RPC with high strength are designed. The W/B of RPC beam is $0.18,0.20$, 0.22. And finite element analysis is used to analyze the bending moment, deflection and other bending properties of high strength reinforced RPC beam under initial cracking and yielding. In this paper, the length of all beams are set to $1800 \mathrm{~mm}$, the net span is $1500 \mathrm{~mm}$, the section size is $150 \mathrm{~mm} * 250 \mathrm{~mm}$. In this paper, the design scheme is shown in Table 1, and structural drawing of beam specimen is shown in Figure 1.

\section{Establishment of finite element model}

Due to the difference of the mechanical properties of concrete and reinforcement, the reinforcement and concrete are defined by different elements, and the solution is independent. Concrete adopts solid65 element, longitudinal tensile reinforcement, vertical bars and stirrups were used link 8 units [7]. The separation process is used to simulate the process, which is in accord with the actual stress condition. RPC beam bottom is simulated by $150 \mathrm{~mm} * 100 \mathrm{~mm}$ of rigid plate beam applied surface 
constraint; in order to prevent stress concentration, $150 \mathrm{~mm} * 100 \mathrm{~mm}$ in the simulation of rigid plate three point loading at the applied load on the beam surface. The details of RPC is shown in Table 1,and the structure diagram of the specimen is shown in Fig.1.

Table.1 Details of RPC Specimens

\begin{tabular}{cccccccc}
\hline $\begin{array}{c}\text { Test } \\
\text { sequence }\end{array}$ & $\begin{array}{c}\text { specimen } \\
\text { number }\end{array}$ & $\begin{array}{c}\text { Copper } \\
\text { coated steel } \\
\text { fiber content } \\
\left(\mathrm{Kg} / \mathrm{m}^{3}\right)\end{array}$ & $\begin{array}{c}\text { Polypropylene } \\
\text { fiber content } \\
\left(\mathrm{Kg} / \mathrm{m}^{3}\right)\end{array}$ & $\begin{array}{c}\text { Cube } \\
\text { W/B }\end{array}$ & $\begin{array}{c}\text { compressive } \\
\text { strength } \\
(\mathrm{Mpa})\end{array}$ & $\begin{array}{c}\text { tensile } \\
\text { strength } \\
(\mathrm{Mpa})\end{array}$ & $\begin{array}{c}\text { Modulus of } \\
\text { Elesticity(Mpa) }\end{array}$ \\
\hline 1 & RPC-A18 & 0 & 0 & 0.18 & 120.82 & 7.50 & 43.70 \\
2 & RPC-A20 & 0 & 0 & 0.20 & 112.01 & 6.44 & 40.10 \\
3 & RPC-A22 & 0 & 0 & 0.22 & 107.34 & 6.17 & 38.90 \\
4 & RPC-B18 & 117 & 0 & 0.18 & 134.50 & 13.06 & 46.90 \\
5 & RPC-B20 & 117 & 0 & 0.20 & 123.71 & 11.11 & 43.70 \\
6 & RPC-B22 & 117 & 0 & 0.22 & 119.73 & 10.06 & 41.00 \\
7 & RPC-C18 & 117 & 0.91 & 0.18 & 125.24 & 11.83 & 45.30 \\
8 & RPC-C20 & 117 & 0.91 & 0.20 & 122.97 & 11.39 & 43.20 \\
9 & RPC-C22 & 117 & 0.91 & 0.22 & 118.33 & 9.78 & 40.20 \\
10 & RPC-D18 & 0 & 0.91 & 0.18 & 118.30 & 6.94 & 42.00 \\
11 & RPC-D20 & 0 & 0.91 & 0.20 & 110.22 & 5.89 & 39.30 \\
12 & RPC-D22 & 0 & 0.91 & 0.22 & 106.80 & 5.44 & 36.40 \\
\hline
\end{tabular}

1 ) RPC : Reactive powder concrete beam;

2 ) A、B、C、D : Fiber type and amount;In turn, it is shown that it is a kind of non - doped fiber, copper - coated steel fiber with $117 \mathrm{~kg} / \mathrm{m}^{3}$, copper - coated steel fiber with $117 \mathrm{~kg} / \mathrm{m}^{3}$ and polypropylene fiber with $0.91 \mathrm{~kg} / \mathrm{m}^{3}$, polypropylene fiber with $0.91 \mathrm{~kg} / \mathrm{m}^{3}$.

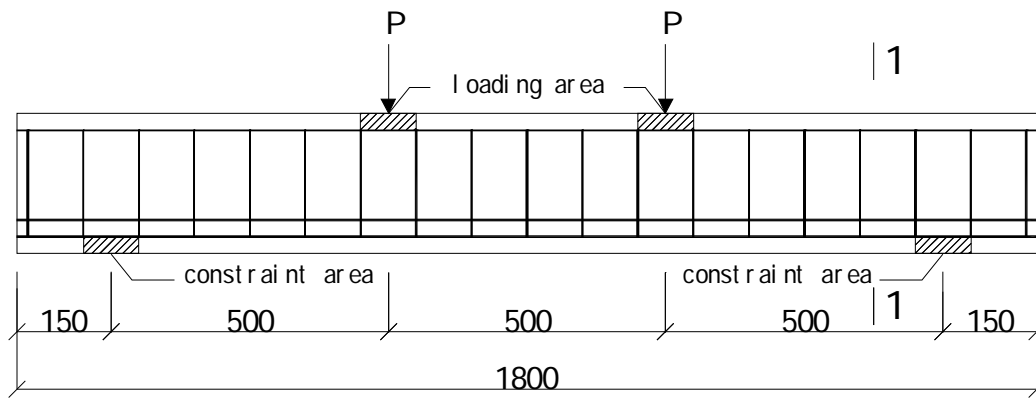
2B 10

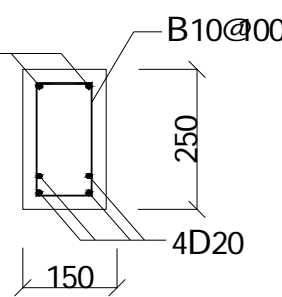

1- 1

Fig.1 The structure diagram of the specimen ( unit : mm )

Poisson's ratio stress relation of RPC is $\sigma=4544.7 v-885.53$, and $\sigma$ is the compressive stress [8]. Assume the Poisson's ratio of RPC is 0.20 . In the finite element simulation analysis, the bilinear model (BKIN) is used in the beam, and the von Mises yield criterion is used. The beam longitudinal reinforcement, Poisson vertical reinforcement and stirrup ratio are 0.30 . When solving the nonlinear problem of reinforced concrete by finite element method, the program is easy to convergence before cracking, cracking as the load increases, the convergence of the program becomes more and more difficult, it is sub grid density, the number of steps, affect the convergence criteria and convergence precision[9]. Therefore, in the parameter design, the shear transfer coefficient of the concrete open crack is 0.5 , the shear transfer coefficient of the closed crack is taken as 0.95 , and the uniaxial compressive strength is -1 , which is means close crushing function. The iteration number of each load step is 60 , and the output frequency is write every substep. The maximum cycle number is in the solution control. The displacement convergence criterion is adopted in the solution process, and the convergence precision is $1.5 \%$. 


\section{Finite element method result and analysis}

Mid-span Bending Moment of High Strength Reinforced RPC Beam in the Initial Cracking Conditions. Figure 2 shows the mid-span bending moment of high strength reinforced RPC beam with different fiber combinations at different W/B values in initial cracking conditions. It can be obtained from Figure 2 that the mid-span bending moment increases with the decrease of the W/B values at the same kind of fiber combination. It can be seen that the decrease of the concrete porosity which is result from the decrease of the W/B values, leading to the RPC beam with a higher strength and a lower mid-span bending moment. In addition, the mid-span bending moment of the RPC beam in initial cracking conditions at the same W/B value and different fiber combinations reaches maximum in group $\mathrm{B}$ and decrease with the order of group C, group A, group D.

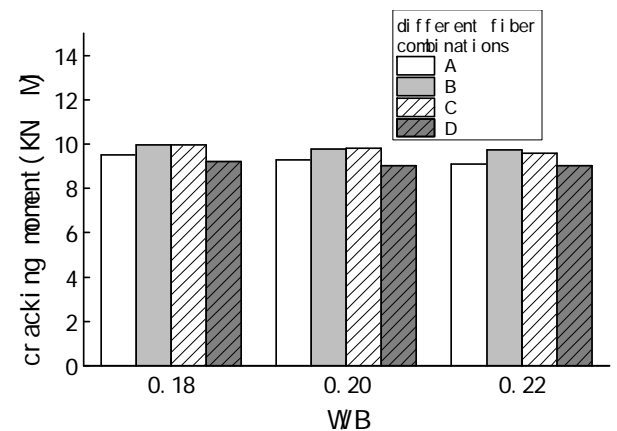

Fig.2 The initial crack midspan moment of RPC beam

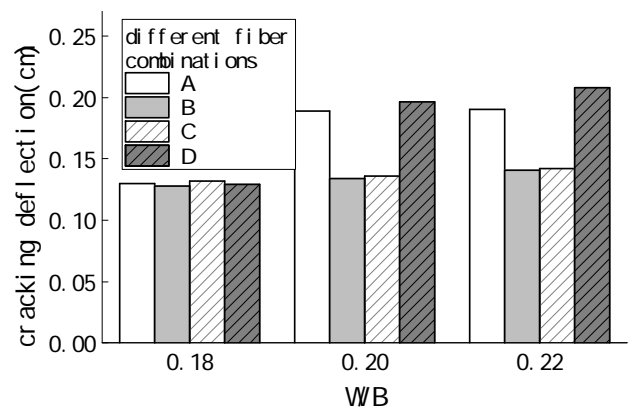

Fig.3 The initial crack deflection of RPC beam

Mid-span Deflection of High Strength Reinforced RPC Beam in the Initial Cracking Conditions. Figure 3 shows the mid-span deflection of high strength reinforced RPC beam with different fiber combinations at different W/B values in initial cracking conditions. It can be seen that the mid-span deflection of the RPC beam in initial cracking conditions increases with the increase of the W/B values at the same kind of fiber combination. When the W/B value amounts to 0.20 , mid-span deflection of the beam for each group drops considerably. The reason for this is that the smaller fiber diameter which makes the increase of the specific surface area requires a larger amount of slurry and the increase of the water requirement, leading to the increase of the reduced amplitude of the mid-span deflection compared to the condition of W/B at the value of 0.18 , although the lower W/B value can raise the strength of the concrete. When the W/B reaches 0.22 , the reduced amplitude of the mid-span deflection is lower than that of the W/B value is 0.20 . It is owing to the effect of the reduced amplitude of the concrete is more obvious than that of composite fiber. Thus, in order to increase the bending performance of the RPC beam, the effect of different W/B values and fiber combinations on the mid-span deflection of the RPC beam needs to be considered.

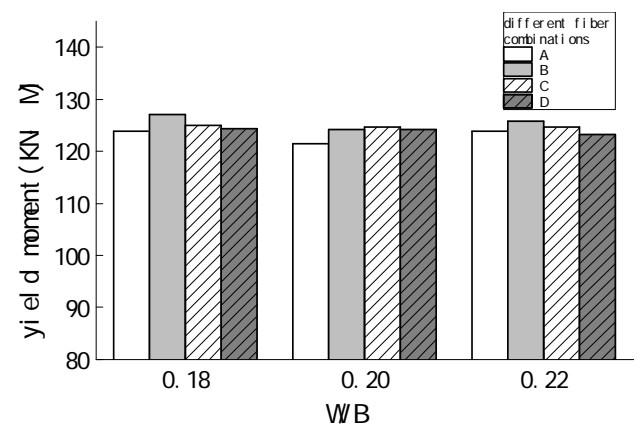

Fig.4 The yield moment of RPC beam

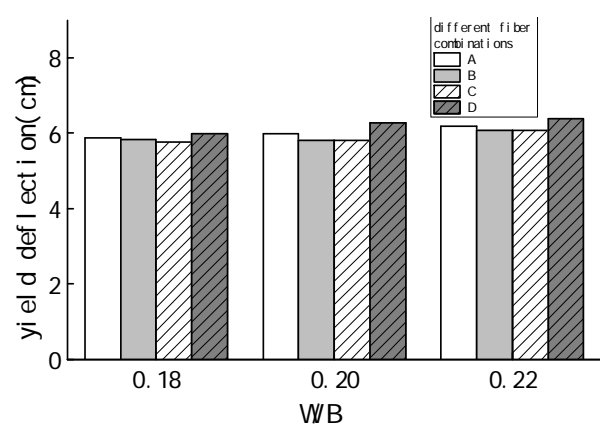

Fig.5 The yield deflection of RPC beam

Mid-span Moment of High Strength Reinforced RPC Beam in Yield Condition. Figure 4 shows the mid-span bending moment of high strength reinforced RPC beam with different fiber combinations at different W/B values in yield conditions. From Figure 4, it can be seen that the mid-span bending moment increases with the decrease of the W/B values at the same kind of fiber combination in yield conditions. The decrease of the bending moment is not very obvious so it can be 
concluded that the value of W/B has no significant influence on the bending performance of the RPC beam in yield conditions. Moreover, the mid-span bending moment of the RPC beam in yield conditions at the same W/B value and different fiber combinations reaches maximum in group $\mathrm{B}$ and decrease with the order of group C, group A, group D.

Mid-span Deflection of High Strength Reinforced RPC Beam in Yield Condition. Figure 5 shows the mid-span deflection of high strength reinforced RPC beam with different fiber combinations at different W/B values in yield conditions. From Figure 5, it can be seen that the mid-span deflection increases with the decrease of the W/B values at the same kind of fiber combination in yield conditions. The mid-span deflection of the RPC beam in yield conditions at the same W/B value and different fiber combinations reaches maximum in group B and decrease with the order of group C, group A, group D.

According to the analysis of flexural behavior of high strength steel reinforced RPC beam, the stress of the cross section is transferred along the fiber after the RPC is added into the steel fiber. The interfacial bonding is transferred to the fiber by the RPC matrix before the high strength RPC beams are cracked. When the cracking occurs, the load at the fracture site is assumed by the fiber, and the interfacial bonding is transferred to the matrix of the cracked cement. And due to the elastic modulus of the steel fiber is higher than RPC, and both of them are mixed to form a composite material with high elastic modulus, which can give full play to the effect of the RPC beam, and has the effect of delaying and preventing the development of cracks, increasing the ultimate bearing capacity and strengthening the flexural performance of the beams. However, the low elastic modulus of polypropylene fiber and RPC composite will reduce the overall elastic modulus, and because of the mechanical properties of RPC itself is very high, far beyond the contribution of polypropylene fiber to make up the internal defects of RPC. Polypropylene fiber in RPC is difficult to form a dense and integrity of the spatial network structure, even will have many weak links. To a certain extent, it reduced the performance of the RPC beam. Therefore group B of RPC only steel fiber beam performances good in the initial cracking and yielding state, followed by the group $\mathrm{C}$ of mixed fine steel fiber and polypropylene fiber, next is group A without fiber, The worst flexural performance is group D only with polypropylene fiber.

\section{Simulation Formula of Normal Section Bending Capacity of High Strength Reinforced Bar RPC Beam}

Ordinary concrete flexural members in the calculation of normal section bearing capacity, because of the influence of the tensile zone of concrete to destroy the bending moment is no more than $1.5 \%$, thus can be neglected[10]. However, high strength reinforced bar RPC concrete beam, as a result of the RPC itself has high tensile strength, so the cracking section of the crack tip to the neutral axis tensile stress is larger, and cracking section of the RPC will exist tensile stress because of the mixed fiber. Therefore, in the calculation of the normal bearing capacity of the high strength reinforced bar RPC beam, it is necessary to consider the influence of the tension zone in the RPC. The principle of replacement in calculation is that the equivalent rectangular stress pattern is equal to the stress of RPC in the actual stress curve and the joint action point of the stress remains the same.

Equivalent Stress Diagram of Tension Zone in Normal Section of RPC Beam. The simplified calculation method of the compressive stress curve on the cross section of the high strength reinforced bar is replaced by the equivalent rectangular stress pattern. The bending moment of RPC beam in compression zone is equation 1(Eq.1)

$$
M_{c}=C\left(\mathrm{y}_{c}+h_{0}-x_{c}\right)=k_{1} f_{c} x_{c} b\left[h_{0}-\left(1-k_{2}\right) x_{c}\right]=\alpha_{1} f_{c} b x\left(h_{0}-\mathrm{x} / 2\right)
$$

Where $y c$ distance from the center of gravity to the edge of the concrete compression zone

$$
C=k_{1} f_{c} x_{c} b=\alpha_{1} f_{c} b x
$$




$$
\mathrm{y}_{c}+h_{0}-x_{c}=h_{0}-\left(1-k_{2}\right) x_{c}=h_{0}-\frac{x}{2}
$$

Assume $\beta_{1}=\frac{x}{x_{c}}=2\left(1-k_{2}\right)$, then $\alpha_{1}=\frac{k_{1}}{\beta_{1}}=\frac{k_{1}}{2\left(1-k_{2}\right)}$. So that the equivalent rectangular stress pattern is determined by the parameters and which called the equivalent rectangular stress coefficient. This paper takes the equivalent rectangular stress pattern coefficient of the compressive zone as $\alpha_{1}=0.89, \beta_{1}=0.75$.

Equivalent stress diagram of normal section compression zone of RPC Beam. The actual stress distribution in the tensile zone of RPC beam with high strength steel bars is a curve. To simplify the calculation and consider the influence of plastic development, Suppose that it is a rectangular tensile stress field with height of $\gamma_{1} \gamma_{2} k \cdot f_{t}$

$$
x_{t}=h-x_{c}=h-x / \beta_{1}
$$

The part below the RPC beam is the height of the tensile zone

$$
\begin{aligned}
& \alpha_{1} f_{c} b x=f_{y} A_{s}+\gamma_{1} \gamma_{2} k \cdot f_{t} b x_{t} \\
& M_{u}=\alpha_{1} f_{c} b x\left(h_{0}-0.5 x\right)-\gamma_{1} \gamma_{2} k \cdot f_{t} b x_{t}\left(0.5 x_{t}-a_{s}\right)
\end{aligned}
$$

where $f y$ the yield strength of tensile steel, $f c$ RPC compressive strength, $f t$ RPC tensile strength, $a_{s}$ the distance between the resultant point and fringe for tensile steel, $A_{s}$ the area of tensile steel, $K$ coefficient of equivalent rectangle distribution of tensile region, $\gamma_{1}$ steel fiber influence coefficient, and the value is 1.0 when no steel fiber, $\gamma_{2}$ polypropylene fiber influence coefficient, and the value is 1.0 when no polypropylene fiber.

Eq. 5 and Eq. 6 is the formulation of the bearing capacity of RPC beam.

Combining the Eq.4, Eq.5and Eq.6, and then obtain the height of compressive region $\mathrm{x}$ is Eq.7

$$
x=\frac{-B+\sqrt{B^{2}-4 A C}}{2 A}
$$

Where the $M_{u}^{t}$ is the simulated breaking bending moment of beam.

$$
A=\frac{1}{2 \beta_{1}} \alpha_{1} f_{c} b-\frac{\alpha_{1} f_{c} b}{2} \quad B=\frac{\alpha_{1} f_{c} b h}{2}-\frac{1}{2 \beta_{1}} f_{y} A_{s} \quad C=\frac{1}{2} f_{y} A_{s} h-f_{y} A_{s} a_{s}-M_{u}^{t}
$$

The $\mathrm{x}$ value of the RPC beam is calculated by the simulation analysis, and bringing it to group A, group $\mathrm{C}$ and group $\mathrm{D}$. Then obtain the value of $\mathrm{k}$ is respectively $0.81,0.91 、 1.17$, the value of $\gamma_{1} k$ is respectively $0.49 、 0.54 、 0.68$, and the value of $\gamma_{2} k$ is respectively $0.92 、 1.20 、 1.29$. It can be seen that the fiber combination will effect the contribution of tensile zone of concrete to the bearing capacity. To simplify the calculation and combined with the computation results, the value of $\mathrm{k}$ is 0.80 , $\gamma_{1} k$ is $0.45, \gamma_{2} k$ is 0.90 . Thus $\gamma_{1}=0.56, \gamma_{2}=1.13$.

Equation of the bearing capacity of RPC beam. According to the stress distribution of beam and balance condition, the Eq. 8 and Eq.9 for calculating the flexural capacity of the reinforced concrete RPC beam is obtained by the equilibrium condition.

$$
0.9 f_{c} b x=f_{y} A_{s}+\gamma_{1} \gamma_{2} 0.8 f_{t} b x_{t}
$$




$$
M_{u}=0.9 f_{c} b x\left(h_{0}-\frac{x}{2}\right)-\gamma_{1} \gamma_{2} 0.8 f_{t} b x_{t}\left(\frac{x_{t}}{2}-a_{s}\right)
$$

Comparison between the calculated and simulated bearing capacity. Bringing the parameters into Eq.8 and Eq.9, and obtain the bearing capacity Error! Reference source not found., then compare with the bearing capacity Error! Reference source not found. that obtained through finite element analysis. The results shows that the average value of Error! Reference source not found./Error! Reference source not found. is 0.978 , standard deviation is 0.018 , and the variable coefficient is 0.018 , which verified the effectiveness and accuracy of the derived formulation.

\section{Conclusions}

Based on the finite element ANSYS simulation, analyze the influence of the initial moment and deflection of the RPC beam on the moment and deflection in the mid span with different fiber combinations and different W/B. The conclusions are as follows:

1) In the initial cracking state, under the same fiber combination, the mid span moment of RPC beam decreases with the increase of $\mathrm{w} / \mathrm{b}$, while the deflection increases with the increase of W/B. Under the action of the same W/B, the best performance of the RPC beams with high strength steel bar is the B group, and the order of the $\mathrm{C}$ group, A group, D group decreased gradually.

2) In yield condition, under the same fiber combination, the mid span bending moment of RPC beam decreases with the increase of $\mathrm{W} / \mathrm{B}$, but the change is not significant. While the deflection increases with the increase of W/B. Copper coated fine steel fiber has a positive contribution to the flexural performance of RPC beams, but Polypropylene fiber is a negative contribution to it.

3) Considering the contribution of RPC beam to the bearing capacity, propose the calculation formula of flexural capacity of normal section of RPC beams under different fiber combinations, The formula is relatively accurate. If you follow the "checklist" your paper will conform to the requirements of the publisher and facilitate a problem-free publication process.

\section{References}

[1] Cheyrezy M, Maret V, Frouin L: Cement \& Concrete Research (1995).

[2] Xiaofei Wang, Yangping Wang:Journal of building materials (2015). in chinese.

[3] Tengfei Ji, Congqi Fang, Chi Li.:Concrete (2016). in chinese.

[4] Xia Cao, Yayun Song, Wei Li, Aihua Tang, Jincheng Peng:Experimental study on basic mechanical properties of single kind steel fiber and polypropylene fiber RPC[A], The twenty-third National Conference on Structural Engineering (Volume III) [C](2014). in chinese.

[5] Haiyan Yuan, Mingzhe An, Fangfang Jia, Chunmei Zhu: Journal of Guangxi University( Nat Sci Ed) (2015). in chinese.

[6] Yang I H, Joh C, Kim B S: Procedia Engineering (2011).

[7] HaiLong Zhang, ChangChun Pei: Applied Mechanics and Materials (2015).

[8] Wenjun Qu, Shengji Wu, Yuhang Qin: Journal of Architecture and Civil Engineering (2008). in chinese.

[9] Pengyong Sun, Shiyong Jiang, Wei Fei, Yi Gan, Binghong Li: Concrete (2008). in chinese.

[10] Li Li. Mechanical behavior and design method for reactive powder concrete beams[D], Harbin Institute of Technology,2010.Haiyan Yuan, Mingzhe An, Fangfang Jia, Chunmei Zhu: Journal of Guangxi University( Nat Sci Ed) (2015).in chinese. 
